\title{
A Tutorial: Applications of Clinical Self-Efficacy Principles to Mentoring of SLP Assistants
}

\author{
Rubini Pasupathy, Renee Bogschutz, Rajinder Koul \\ Texas Tech University Health Sciences Center, Lubbock, TX, USA
}

There are an increasing number of speech-language pathology assistants (SLP-As) in the public school settings under the supervision of speech-language pathologists (SLPS) in the United States. One of the functions of SLPS is to mentor and facilitate the development of SLP-As. The primary aim of this tutorial is to use the social cognitive model of self-efficacy to enhance the knowledge and skills of SLP-As. Literature in social cognitive theory indicates that self-efficacy is a predictor of performance, particularly in education and health domains. Effectively incorporating the self-efficacy framework in the mentoring of SLP-As will improve clinical performance and outcomes, as well as increase job satisfaction and retention of SLPAs in the school setting.

Keywords: Clinical self-efficacy, Mentoring, Speech-language pathology assistants, Public schools

\section{INTRODUCTION}

The services of Speech-Language Pathologists (SLPs) are in high demand in the United States. The educational requirements of SLPs in the United States are rigorous and include a minimum of a master's degree in speech-language pathology or communication disorders (American Speech-Language-Hearing Association [ASHA], 2014). Since the 1970s Speech-Language Pathology Assistants (SLP-As) have been serving as support personnel for SLPs, primarily in school settings. The basic educational requirements to qualify to be an SLP-A are an associate's or bachelor's degree in speech-language pathology or communication disorders, and a minimum of a hundred hours of field or clinical work experiences under the supervision of qualified SLPs (American Speech-Language-Hearing Association [ASHA], 2013). SLP-As expand the range of practice of speech-language pathology by either increasing the frequency or intensity of services provided. According to the American Speech-Language-Hearing Association, the primary functions of SLP-As include service delivery, administrative support, and prevention advocacy. The specific tasks included in these services range from assisting the SLP during assessment, following documented treatment plans, recording client performance, assisting with clerical duties, and supporting the SLP in research projects, training, and public relations programs. By performing these daily functional activities SLP-As allow SLPs to concentrate on the professional level clinical activities that require clinical judgment (ASHA).

SLPs play an important role in supervising and mentoring SLP-As. SLP-As work in

(C) 2017 The Korean Association of SpeechLanguage Pathologists

This is an Open Access article distributed under the terms of the Creative Commons Attribution Non-Commercial License (http:// creativecommons.org/licenses/by-nc/4.0/) which permits unrestricted non-commercial use, distribution, and reproduction in any medium, provided the original work is properly cited. 
school settings with only basic speech-language pathology education and minimal clinical experience. However, clinical outcomes are highly dependent on SLP-As' knowledge and skills. The provision of effective therapy requires SLP-As to be conscious of and modify their behavior to meet the needs of students they serve in the school setting. ASHA suggests that the training of supervisors can be further improved (ASHA, 2008). According to ASHA (2008) effective supervision of SLPAs should include activities that involve self-analysis and selfevaluation. Therefore it is important for supervising SLPs to incorporate measures that allow SLP-As to assess and build confidence in their therapy skills to ensure that they are successful.

SLP supervision and mentoring are especially beneficial for inexperienced SLP-As who have the complex task of putting into practice the knowledge and skills acquired as undergraduate students. Self-efficacy beliefs influence performance and behavioral change, especially in the beginning stages of skill acquisition. The primary goal of this tutorial is to present a comprehensive overview of the self-efficacy framework and to demonstrate the application and value of the self-efficacy framework to the training and mentoring of SLP-As throughout their career.

\section{THEORETICAL OVERVIEW}

According to Bandura (2006), individuals shape their life circumstances by intentionality, forethought, self-regulation, and self-reflection. Individuals take actions based on forethought or visualized futures that involve the formulation of action plans and strategies (Bandura, 2006). To attain these visualized futures or goals individuals self-regulate and take appropriate actions while constantly self-examining and making corrective adjustments. This self-examination involves the judgment of personal self-efficacy (Bandura, 2006). Humans are able to reflect and evaluate their experiences, thought processes and environments (Pajares, 1996). Human agency does not occur in a vacuum; it is influenced by the triadic interaction between cognitive, behavioral, and environmental factors (Bandura, 1986, 2006). In acting as agents for themselves individuals make causal attributions that shape their life circumstances. Bandura (1997) believes that behavior is a product of cognitive self-influence.

According to Bandura (1997), self-efficacy is the core belief that enables human agency, or the ability of individuals to act intentionally with forethought and self-regulation. The belief that one is capable of shaping and influencing his/her life circumstances and envisioned future enables human agency. In the absence of self-efficacy there is little incentive or motivation to pursue goals and persevere through difficulties (Bandura, 2006).

\section{WHAT IS SELF-EFFICACY}

Perceived self-efficacy refers to "beliefs in one's capabilities to organize and execute the courses of action required to produce given attainments" (Bandura, 1997, p. 3). Self-efficacy should not be confused with other self-beliefs. It is important that self-efficacy is distinguished from self-beliefs such as selfconcept and self-esteem. Self-concept, or the sum of selfknowledge one holds of oneself, differs from self-esteem, which is a judgment of worthiness. These two concepts differ from self-efficacy, which is defined as the judgment of confidence in one's capabilities (Pajares \& Schunk, 2001). According to Pajares and Schunk (2001), self-concept and self-esteem are not related to self-efficacy. An SLP-A may have a very negative perception of self and have very low self-esteem, but at the same time be very confident about his or her speechlanguage pathology clinical capabilities.

While self-concept and self-esteem are global perceptions of oneself, self-efficacy is context specific (Bong, 2006). Selfefficacy beliefs are perceptions of confidence in the ability to perform a specific task and vary according to task generality (Bandura, 1977). Therefore, it is possible for an SLP-A to be very confident about his/her ability to pass an examination in an undergraduate articulation and phonology course, but at the same time, not be confident about providing articulation therapy. Among clinical tasks, an SLP-A may be highly confident about providing therapy to students within the public school setting, but may be less confident about his or her ability to provide therapy to students with developmental disabilities, especially as an inexperienced SLP-A.

Self-efficacy beliefs also vary on two other dimensions: magnitude and strength (Bandura, 1977). Self-efficacy beliefs differ according to the magnitude or difficulty level of tasks. For example, SLP-As may be highly confident about their abilities to conduct an individual therapy session with a compliant student, but may not be confident about their ability to conduct group therapy with students who demonstrate defiance and behavioral disruptions. The strength of efficacy beliefs also differs for various tasks. Individuals can either have strong self-beliefs of their capabilities that withstand setbacks 
and failures, or have weak self-beliefs that disintegrate in the face of minor adversities. Although an SLP-A may be confident about conducting therapy, these self-beliefs may be weak and may not endure minor setbacks, such as criticisms from a student's parents. However, an SLP-A with strong selfefficacy beliefs will perceive and cognitively process negative feedback in a manner that does not influence their confidence in their capability. In the remainder of this section, the predictive nature, mediating processes, and various sources of selfefficacy are discussed.

\section{PREDICTIVE NATURE OF SELF-EFFICACY}

The popularity of the self-efficacy construct is, in part, due to its ability to predict future behavior. Self-efficacy is a predictor of performance (Bandura, 1997). Research indicates that there is a significant correlation between self-efficacy and performance on specific tasks (Bandura, 1982; Bandura \& Adams, 1977). There is a difference in behavior among individuals that have high self-efficacy beliefs and those that do not have such beliefs. Individuals that hold high self-efficacy beliefs have higher aspirations, have strong commitment to their goals, and are not easily discouraged by obstacles and failure (Bandura, 1997).

The effort invested in a task is regulated by an individual's belief in his or her ability to perform the task. Research also indicates that self-efficacy increases persistence in continuing with a task (Bandura, 1977). In a study conducted with 105 undergraduate students, Lent, Brown and Larkin (1986) compared the prediction of grades and persistence of students that were divided into high and low self-efficacy groups based on their self-efficacy scores. Students with high self-efficacy scores remained longer in college, were more persistent $(t=2.35, p<0.05)$, and achieved higher grades $(t=1.97$, $p<0.05)$. According to Bandura (1977), individuals with low self-efficacy tend to invest less effort in succeeding in a specific task than those with high self-efficacy beliefs. Bandura believes that people invest effort in an activity based on their expected outcome of that activity. When an individual has low self-efficacy and believes that the successful completion of the task is not possible, little effort is invested in the task. These individuals utilize fewer coping strategies and exhibit lower mastery of the tasks, which in turn reinforces their low self-efficacy beliefs. For example, an SLP-A who has high clinical self-efficacy may work hard to solve complex treatment problems and continue to persevere through the various obstacles and setbacks that are commonly encountered in treating students in the school setting. However, an SLP-A with low confidence in his or her clinical skills may choose not to invest too much effort in a venture that is expected to fail.

\section{MEDIATING PROCESSES OF SELF-EFFICACY}

Self-efficacy influences human functioning through the following mediating processes: cognitive, motivational, affective, and selective processes (Bandura, 1997). Self-efficacy beliefs influence cognitive processes, such as forethought and goal setting (Bandura, 1997). Envisioned futures are highly dependent on the belief that one is capable of achieving these goals. Locke and Latham (2002) found that the strength of self-efficacy is positively correlated to the goal level chosen. Individuals with high self-efficacy tend to set higher goals as they believe that they are capable of performing the task successfully. Therefore, SLP-As who have high clinical self-efficacy beliefs may strive to further their education and work towards SLP certification. SLP-As who have low perceptions of SLP clinical self-efficacy may not attempt such complex goals.

Setting of goals does not ensure achievement of these goals. Individuals need to be motivated to attain imagined futures. According to Bandura (1997), self-efficacy beliefs influence cognitive regulation of motivation through causal attributions, outcome expectancies, and goals. Personal judgments about the causes of past successes and failures influence motivation and behavior. For example, SLP-As who have high self-efficacy beliefs may attribute their past successes to high ability, and may work hard to attain their goals. However, SLP-As who have low self-efficacy beliefs may attribute their successes to external factors such as luck, and may not be as inspired to achieve a goal that is believed to be unattainable.

According to Bandura (1997), self-efficacy is a mediating factor between self-regulation and emotional states. Individuals who are highly confident of their ability to be successful in a particular task or situation are able to cognitively influence their emotional state. In contrast, individuals who are not confident about their abilities to be successful experience stress and anxiety due to the lack of confidence in the ability to influence their environment and succeed in a particular task. Therefore, an individual's ability to focus on the task at hand is reduced. For example, SLP-As who have low self-efficacy beliefs have to not only overcome clinical obstacles, but also expend effort to overcome the obstacles of stress and anxiety.

Lastly, self-efficacy is an intervening factor in life decisions 
because it influences selection processes (Bandura, 1997). Life is shaped by choices and decisions. Individuals have a certain degree of control over their environment and behavior. Bandura (1997) states that the choices individuals make are influenced by their self-efficacy beliefs, including preferences for graduate education and job selection. Lent, Brown and Hackett (1994) and Hackett and Betz (1981) state that career choices are influenced by self-efficacy beliefs. Wood and Bandura (1989) conclude that a strong sense of self-efficacy is integral to decision-making as it is easy to shift the focus from the task at hand to one's own capabilities, or lack thereof. In organizational situations that are difficult or when failure is imminent, it is important that one has a strong sense of selfefficacy. Wood and Bandura conclude that a strong sense of self-efficacy enables a decision maker to focus his or her cognitive capabilities on the task at hand and not waste limited cognitive resources on questioning their capabilities.

\section{SOURCES OF SELF-EFFICACY}

Self-efficacy is developed through the cognitive integration of the following four information cues: enactive mastery, vicarious experience, verbal persuasion, and emotional arousal (Bandura, 1982). Enactive experiences influence self-efficacy through the cognitive processing of informational cues about successes and failures. Vicarious experiences provide additional information on capabilities through the comparison of an individual's perceived capabilities with the achievements of others (Bandura, 1997). Verbal judgments or feedback and emotional and physiological reactions influence self-beliefs by providing further information about perceived capabilities (Bandura, 1997).

\section{Enactive experiences}

Enactive experiences are the most influential in the development of self-efficacy (Gist, 1987) because enactive experiences contain the most authentic evidence of capabilities (Bandura, 1997; Usher \& Pajares, 2008). Self-efficacy is most influenced as an individual is first introduced to the task and begins skill development (Bandura, 1982; Usher \& Pajares, 2008). The frequent, successful completion of a specific task increases selfefficacy (Bandura, 1982). Individuals gain confidence in their ability to complete a specific task when they are repeatedly successful in completing the task. In contrast, repeated failures may erode self-efficacy beliefs (Gist, 1987). For example, SLP-As who have had successful clinical outcomes may de- velop high clinical self-efficacy, whereas SLP-As who have received multiple complaints from students' parents may begin to doubt their capabilities and have low clinical self-efficacy.

According to Locke (2000), enactive mastery occurs through a progression of three phases. The first phase includes the introduction and exhibition of skills to master a task. The second phase incorporates opportunities for individuals to master the skills through guided practice and simulated conditions, and the third and final phase involves the provision of progressive opportunities to conduct the task at various difficulty levels. Locke states that attainment of sub-goals enhances self-mastery and challenging sub-goals enhances perceived self-efficacy.

Within enactive experiences there are several factors such as effort, task difficulty, and performance attribution that influence self-efficacy beliefs. According to Bandura (1997), a high level of effort invested in a task negatively influences selfefficacy belief, as the individual perceives that he or she does not have the appropriate abilities to perform the task successfully with minimal effort. Unsuccessful outcomes that involve high effort can decrease self-efficacy, whereas successful outcomes that entail little effort may not influence self-efficacy positively. Enactive experiences are even more influential on self-efficacy when the task is challenging and obstacles are surmounted (Bandura, 1997).

Bandura (1977) states that self-efficacy is affected by the attribution of causes of failure or success. He explains that attributing success to external factors can diminish self-efficacy; whereas, attribution to internal factors tends to increase selfefficacy. For example, an SLP-A's clinical self-efficacy may not increase if positive clinical outcomes are attributed to external factors such as easy cases or luck. Conversely, a bad clinical outcome perceived as a result of poor therapy skills could jeopardize an SLP-A's clinical self-efficacy. According to Gist (1987) and Gist and Mitchell (1992), the strength of self-efficacy beliefs is a mediating factor in the interpretation and attribution of success and failures of tasks. Individuals who are highly confident about their abilities are more resistant to failures, as these individuals tend to attribute failure to external unpredictable factors such as chance or random occurrence. Whereas, the efficacy levels of individuals with low self-efficacy are easily influenced by failures as they tend to attribute their failures to internal stable factors, such as ability.

\section{Vicarious experiences}

Vicarious experiences or modeled performances are the sec- 
ond most influential variable in the development of self-efficacy (Bandura, 1997; Gist, 1987). Individuals use social models as benchmarks to form judgments of their own capabilities (Bandura, 1997; Usher \& Pajares, 2008). Individuals assess their capabilities by observing the performance of others and comparing their accomplishments with that of others (Usher \& Pajares). According to Carroll and Bandura (1987), individuals observe, compare and make judgments about their skill and capabilities through a process called conception matching. Individuals observe successful behaviors and make judgments about their capabilities by comparing their skills with those of successful models (Gist \& Mitchell, 1992).

There are several conditions when self-efficacy is more influenced by vicarious experience. These conditions include: uncertainty about capabilities, similarity between individual and model, modeling of coping strategies, and competent models. According to Bandura (1997), self-efficacy is highly influenced by vicarious experience, especially when individuals are uncertain about their capabilities and do not have the relevant experiences to form judgments. For example, modeling effective therapy behaviors by successful SLPs may highly influence the self-efficacy of SLP-As who have yet to form judgments of their own capabilities.

The effect of vicarious experiences on self-efficacy is enhanced when there are similarities between individuals and those performing the tasks (Bandura, 1977; Wood \& Bandura, 1989). Modeling is most influential on self-efficacy when there is a high degree of similarity between the model and individual, especially in age and gender (Bandura, 1997). Therefore the clinical self-efficacy of young female SLP-As may be positively influenced by the observation of successful female SLP role models.

Models who exemplify coping strategies have a strong effect on self-efficacy (Bandura, 1997). According to Bandura (1997), models who endure setbacks and exhibit coping strategies convey the primacy of effort over ability. The demonstration of perseverance encourages self-efficacy beliefs. For example, observation of a successful SLP who demonstrates coping strategies and persists to obtain a successful outcome with a student following an initial period of struggle will heighten the clinical self-efficacy beliefs of the SLP-A.

Competent models strengthen the self-efficacy of individuals more than incompetent models (Bandura, 1997). Individuals selectively observe and pay attention to competent models because they hope to learn and imitate the success of the models (Bandura, 1986). In the school setting, inexperienced
SLP-As should seek competent SLP-As and SLPs from within the school district to serve as models and mentors.

\section{Verbal persuasion}

The overt and covert messages that an individual receives from significant others such as peers, parents, spouses, teachers, and SLP supervisors may influence the perceptions of his or her performance capabilities (Gist, 1987). Bandura (1997) referred to this source of self-efficacy information as verbal persuasion. Evaluative feedback on performance is an example of verbal persuasion (Bandura, 1997). Positive verbal persuasion encourages individuals to attempt, persist, and succeed, which in turn promotes skill development and enhances self-efficacy beliefs (Bandura, 1997). However, there is also the danger of undermining self-efficacy through insincere positive feedback (Bandura, 1997). Individuals who are falsely praised for their skills quickly realize the deceit through experience and failure, which may further undermine their confidence. Negative verbal persuasion can lower self-efficacy by inhibiting individuals and preventing them from attempting a particular task. Pajares (2002) cautions that negative verbal persuasion, such as negative feedback, can influence selfefficacy more than positive encouragement. The framing of evaluative feedback also plays a role in influencing self-efficacy. Schunk (1984) reports that feedback that highlights capabilities instead of effort and hard work, especially in the early phases of skill development, promotes self-efficacy.

Although verbal persuasion is a variable that contributes to self-efficacy, its effects are not as strong as enactive mastery and vicarious learning. This is because the effect of verbal persuasion on self-efficacy is mediated by multiple factors (Bandura, 1982). The effectiveness of verbal persuasion is affected by existing self-beliefs, credibility of the source, and familiarity of the source with task demands (Bandura, 1982). Verbal persuasion is most effective when individuals have certain existing beliefs of their capabilities (Bandura, 1997). Therefore, positive messages may increase the self-efficacy of those who are confident of their capabilities and negative messages can reduce the self-efficacy of individuals who are unsure of their capabilities. Verbal persuasion is only effective if an individual perceives that the person providing the feedback or persuasion is knowledgeable and has expert knowledge of the particular task. For example, feedback from a nonclinician such as a teacher or principal may not impact the clinical self-efficacy of an SLP-A. However, feedback from a highly skilled SLP may influence the clinical self-efficacy of 
the recipient, especially if the recipient holds similar beliefs about his or her capabilities.

\section{Physiological and affective states}

The final source of self-efficacy information is emotional and physiological arousal. Physiological states, such as strength, stamina, aches and pains, and mood affect self-efficacy judgments (Bandura, 1997). According to Bandura (1997), more than the mere presence of these somatic states, the manner in which individuals perceive and interpret physical and emotional arousal is influential on their efficacy beliefs. An individual who is highly confident of his or her capabilities will interpret his or her somatic arousal in a positive manner, such as excitement, and may not attend to any aches or pains while performing a particular task. This interpretation facilitates the successful completion of the task (Bandura, 1997). However, individuals with low confidence in their abilities may perceive the presence of a headache or stomachache as indicators of stress or anxiety and cognitively assimilate the message that competence on the particular task or skill is lacking. These interpretations may inhibit optimal performance and further reinforce the low efficacy expectations. Therefore the cognitive processing of emotional and physiological arousal influences self-efficacy beliefs (Bandura, 1997).

\section{SLPS' CLINICAL SELF-EFFICACY BELIEFS}

Clinical self-efficacy has not been widely studied in speechlanguage pathology. There are only three published studies that have investigated SLPs' clinical self-efficacy. Rudolf, Manning and Sewell (1983) investigated clinical self-efficacy of students who were treating persons with fluency disorders. They observed that clinical self-efficacy of speech-language pathology students increased with enactive mastery. Enactive experiences influence self-efficacy through the cognitive processing of informational cues about successes and failures (Bandura, 1997). In another study, Lee and Schmaman (1987) examined the relationship between graduate clinical experience and SLPs' clinical self-efficacy. Results indicated that SLPs' clinical self-efficacy increased after the first academic year of clinical experience. A significant positive relationship was reported between SLPs' clinical self-efficacy and clinic performance as judged by clinical supervisors $(\mathrm{r}=0.36$, $p<0.05$ ). These results were further substantiated by a study conducted on speech-language pathology graduate students by Pasupathy and Bogschutz (2013). Pasupathy and Bog- schutz report that graduate students with greater hours of clinical experience are more confident about their ability to complete clinical tasks successfully than graduate students with fewer hours of clinical experience. A significant positive relationship $(\mathrm{r}=0.51, p<0.01)$ was reported between speechlanguage pathology graduate students' clinical self-efficacy beliefs and clinical performance as judged by clinical supervisors. Further, Pasupathy and Bogschutz, observed wide variability in speech-language pathology graduate students' clinical self-efficacy beliefs across clinical tasks domains and report a significant main effect for speech-language pathology clinical task domain, $\mathrm{F}(6,398)=62.31, p<0.01$ and report that year of graduate study (i.e., first year versus second year) had a significant effect $\mathrm{F}(7,40)=4.16, p<0.01$ on speech-language pathology graduate students' clinical task domain. Overall, graduate students were more confident about completing speech-language pathology clinical tasks that were related to communicating with clients, and were least confident about completing speech-language pathology clinical tasks that were related to the evaluation of clients. According to Cecil \& Pinkerton (2000) self-efficacy beliefs are reported to vary according to the magnitude and difficulty level of tasks. Research indicates that SLPs' clinical self-efficacy plays a key role in clinical performance. Therefore, SLP-As may benefit from strategies that enhance speech-language pathology clinical self-efficacy.

\section{STRATEGIES TO ENHANCE SLP-AS' CLINICAL SELF-EFFICACY BELIEFS}

This section proposes specific strategies to enhance the clinical self-efficacy of SLP-As. In many school settings, meticulous SLPs are already using many of the strategies presented here. The self-efficacy framework, however, provides a theoretical model for SLPs to use when supervising and mentoring SLPAs. In addition, the self-efficacy framework highlights the importance of particular strategies that may not be employed effectively when supervising and mentoring SLP-As in school settings. For example, SLPs may not provide task specific feedback to SLP-As.

To enhance SLP-As' clinical self-efficacy beliefs through mastery experiences, the SLP can implement the following strategies when supervising and mentoring assistants:

- Consider case difficulty and skill development when assigning cases to foster initial success. The SLP should be cognizant of the SLP-A's skill level, especially at the begin- 
ning of his/her career, and assign cases or tasks that will encourage confidence through successful outcomes. For example, the SLP should initially assign clients who have been previously enrolled in speech-language services and who have demonstrated compliance and successful therapeutic gains. The SLP should avoid initially assigning clients with multiple or complex conditions who have a higher probability of unsuccessful outcomes or disruptive/destructive behavior to inexperienced assistants.

- Introduce complex clinical tasks by breaking down each task into easier sub-skills to encourage success and skill acquisition in order to build confidence. The SLP should be cognizant of the various sub-skills and skill difficulty involved in therapeutic tasks when making assignments. Experienced SLPs may lose sight of the various components involved in daily treatment, whereas SLP-As may need support and guidance through each individual step of the total process. For example, in a reading comprehension task, the SLP should break down the task into the sub skills of selecting appropriate text, providing appropriate reading scaffolding techniques, generating appropriate comprehension questions, and providing appropriate feedback to the student. The SLP should highlight and provide additional support for the sub-skill that is the most challenging, such as selecting an appropriate reading comprehension passage.

- Introduce and encourage the use of concrete rules and strategies to reduce cognitive stress and effort while performing therapeutic tasks. It would be beneficial for the SLP to generate a written list of rules and strategies for a wide variety of conditions, such as behavior management approaches, scaffolding structures, and student feedback scripts, that the SLP-A could refer to and utilize during therapy.

- Be aware of preexisting self-schemata. Explore and discuss with the SLP-A his or her self-perception as a therapist. Encourage the development of positive self-schemata by addressing any cognitive distortions. For example, if an SLP-A has not been successful in gaining admission into graduate school, he or she may perceive this as an indicator of poor clinical skills. The SLP can make clear that theoretical knowledge and clinical abilities are two very different skill sets.

- Be aware of the confusion between effort and ability and the influence on self-efficacy. Explain to the SLP-A that degree of effort invested in a task is not an indicator of ability or outcome. The SLP must make clear that even though a significant amount of effort has been invested in a task, a positive outcome is not a guarantee and confidence in performing the task should not be dependent on effort invested in performing the task. For example an SLP-A may invest significant effort in creating a therapy activity, however it may be unsuccessful if the child is not attracted, interested, or motivated by the activity.

- Encourage the SLP-A to practice newly learned skills at home to reinforce learning. Encourage the SLP-A to roleplay use of therapy skills with family and/or peers in preparation for therapy the next day. This will increase the SLPA's confidence in performing the upcoming therapy tasks successfully.

- Keep in mind that failing to perform a task successfully in a public arena may negatively impact self-efficacy beliefs. Ensure that SLP-As are well prepared for tasks that involve public demonstration or speaking, such as educating the families of students on communication disorders.

To enhance SLP-As' clinical self-efficacy beliefs through $v i$ carious experiences, the SLP can implement the following strategies when supervising and mentoring assistants:

- Create opportunities for the SLP-A to observe effective therapy. The supervising SLP should act as a model and verbalize thought processes and therapeutic strategies aloud. Additionally, videotape and review with the SLP-A successful treatment sessions, as well as encourage the SLP-A to self-model through either videotaping or practicing sessions in front of a mirror.

- The SLP should frequently model coping behaviors and engage in problem solving activities with the SLP-A. For example, the SLP might model behavior management approaches such as redirection, sensory breaks, use of timers, or preferred activities as rewards. Also, encourage the SLP-A to conduct cognitive rehearsal of coping mechanisms at home or with peers.

- Encourage mentoring partnerships that highlight the similarities between the model and the SLP-A. Clinical self-efficacy is most persuasive when there is a degree of similarity between the SLP and the SLP-A. For example, pair a bilingual therapist with a bilingual assistant to enhance the SLP-A's clinical self-efficacy through modeling.

- Create opportunities for groups of SLP-As within a school district to meet and discuss experiences and coping strategies. This will allow SLP-As to recognize that they are not alone in experiencing the various obstacles in clinical skill 
development. Self-efficacy is enhanced via vicarious learning through shared experiences.

- The SLP should acknowledge that social comparison is instinctive during skill acquisition. When an individual is learning new skills, it is only natural for that person to compare their performance with that of others. Self-efficacy is highly dependent on the type of social comparison that is conducted; therefore, SLP-As should be encouraged to compare performance with individuals who are at the same stage of skill development and experience. Conversely, the SLP-A's clinical self-efficacy would decrease if he or she compared his or her performance with that of a highly experienced SLP.

To enhance SLP-As' clinical self-efficacy beliefs through verbal persuasion, the SLP can implement the following strategies when supervising and mentoring assistants:

- To enhance the SLP-A's clinical confidence, the supervising SLP should be cognizant of the framing of feedback. Effective feedback can boost clinical self-efficacy, whereas poorly framed feedback can undermine existing clinical self-efficacy. Feedback regarding successful therapy tasks should be framed in terms of ability rather than effort. For example, the supervising SLP should praise the clinical skillset that led to effective group therapy, rather than highlight the effort invested in the preparation for the therapy session. Feedback about unsuccessful experiences should concentrate on strategies to improve clinical performance, rather than on lack of clinical skills and abilities. The SLP should avoid disparaging feedback about unsuccessful clinical outcomes, as it may reduce the SLPA's clinical self-efficacy and inhibit future attempts and perseverance in challenging clinical tasks.

- The SLP should be cognizant of the impact of social validation and social appraisal on clinical self-efficacy. The supervising SLP can easily transmit negative social validation and appraisal through nonverbal and pragmatic means. For example, an SLP with the intention of building confidence in a poorly performing SLP-A may over praise mediocre clinical outcomes or repeatedly offer unsolicited advice; however, these actions transmit the SLP's underlying low opinion of the SLP-A's skill development. These types of nonverbal or unconscious negative social appraisals can easily damage the SLP-A's clinical self-efficacy.

- Praise from knowledgeable and credible sources increases confidence; however, disingenuous praise negatively influences clinical self-efficacy. The SLP may mistakenly be- lieve that praising all outcomes increases confidence. Instead the SLP should selectively praise specific salient outcomes. For example, avoid careless use of generalized praise such as "good job today;" instead selectively praise execution of skills or tasks that are exceptional such as "you did a good job with redirecting Bobby back to the articulation task this morning."

- Encourage the SLP-A to measure success in terms of selfimprovement over time. For example "today all of the children in group therapy were actively attending to the task, because you used consistent behavior management strategies, which is a great improvement from last week when many of the children were disengaged."

To enhance SLPs' clinical self-efficacy beliefs through awareness of the effect of physiological and affective states on confidence, the SLP can implement the following strategies when supervising and mentoring assistants:

- The supervising SLP should acknowledge that anxiety is a normal response to new skill performance. The SLP-A needs to be aware of the negative effects of anxiety on selfefficacy. Counsel the SLP-A to be conscious of physiological states and self-monitoring these conditions. The SLP should explain to the SLP-A that stress and anxiety is often expressed as body aches and pains, sweating, and/or nausea. Further explain that these physiological expressions of emotions should not be interpreted as indicators of lack of clinical skill, but are just the body's way of dealing with stress.

\section{IMPLICATIONS OF ENHANCED SLP CLINICAL SELF-EFFICACY BELIEFS}

There are several implications to enhancing the SLP clinical self-efficacy of SLP-As practicing in school settings. SLP-As who are confident about their clinical abilities are more likely to provide higher quality therapy, be more engaged, and persist through difficult encounters. Successful therapy outcomes facilitate job satisfaction and promote employee retention. SLP-A job satisfaction and retention is beneficial to the school setting and the profession as a whole. Individuals with high self-efficacy beliefs have high aspirations, have strong commitment to their goals, and are not easily discouraged by obstacles and failure (Bandura, 1977). Further, research indicates that individuals who are highly confident about their abilities continue to persist through obstacles. Therefore, enhancing SLP's clinical self-efficacy may promote retention of 
SLP-As, thus reducing turnover.

According to Bandura (1997) self-efficacy is a predictor a performance; therefore, SLP-As with high SLP clinical self-efficacy will perform clinical tasks more successfully and thereby increase the quality of therapeutic services. The relationship between self-efficacy and performance has been demonstrated in many areas of education. The self-efficacy beliefs of teachers are reported to be a predictor of job satisfaction and student learning outcomes (Capara, Barbaranelli, Steca, \& Malone, 2006). Incorporating, self-efficacy building strategies will facilitate the development of more skillful and confident SLP-As and thereby improve job satisfaction, retention, and clinical outcomes.

\section{REFERENCES}

1. American Speech-Language-Hearing Association. Clinical supervision in speech-language pathology [Internet]. Rockville, MD: American Speech-Language Hearing Association; 2008. Available from: www.asha.org/policy/tr2008-00296.htm

2. American Speech-Language-Hearing Association, Speech-language pathology assistant scope of practice [Internet]. Rockville, MD: American Speech-Language Hearing Association; 2013. Available from: http://www.asha.org/policy.Indexterms:SLPAs,scope

3. American Speech-Language-Hearing Association. Frequently asked questions: Speech-language pathology assistants (SLPAs). [Internet]. Rockville, MD: American Speech-Language Hearing Association; n.d. Available from http://www.asha.org/associates/ SLPA-FAQs/

4. Bandura A. Self-efficacy: Toward a unifying theory of behavioral change. Psychol Rev. 1977;84:191-215.

5. Bandura A. Self-efficacy mechanism in human agency. Am Psychol. 1982;37:122-147.

6. Bandura A. Social foundations of thought and action: A social cognitive theory. Englewood Cliffs, NJ: Prentice-Hall; 1986.

7. Bandura A. Self-efficacy: the exercise of control. New York: W.H. Freeman; 1997.

8. Bandura A. Guide for constructing self-efficacy scales. In Pajares F \& Urdan T, editors. Self-efficacy beliefs of adolescents. Greenwhich, CT: Information Age Publishing; 2006.

9. Bandura A, Adams NE. Analysis of self-efficacy theory of behavioral change. Cognit Ther Res. 1977;1:287-308.

10. Bandura A, Adams NE, Beyer J. Cognitive processes mediating behavioral change. J Pers Soc Psychol. 1977;35(3):125-139.

11. Bong M. Asking the right question: How confident are you that you could successfully perform these tasks? In Pajares F \& Urdan T, editors. Self-efficacy beliefs of adolescents. Greenwhich, CT: Information Age Publishing; 2006.
12. Caprara GV, Barbaranelli C, Steca P, Malone PS. Teachers' self-efficacy beliefs as determinants of job satisfaction and students' academic achievement: A study at the school level. J Sch Psychol, 2006;44(6):473-490.

13. Cecil H, Pinkerton SD. Magnitude : An Important dimension of self-efficacy. J Appl Soc Psychol. 2000;30(6):1243-1267.

14. Carroll WR, Bandura A. Translating cognition into action: The role of visual guidance in observational learning. J Mot Behav. 1987;19: 385-398.

15. Gist ME. Self-efficacy: Implications for organizational behavior and human resource management. Acad Manage Rev. 1987;12(3): 472-485.

16. Gist ME, Mitchell TR. Self-efficacy: A theoretical analysis of its determinants and malleability. Acad Manage J. 1992;17(2):183-211.

17. Hackett G, Betz NE. A self-efficacy approach to the career development of women. J Vocat Behav. 1981;18:326-339.

18. Lee C, Schmaman F. Self-efficacy as a predictor of clinical skills among speech pathology students. High Educ. 1987;16(4):407-416.

19. Lent RW, Brown SD, Hackett G. Toward a unifying social cognitive theory of career and academic interest, choice and performance. J Vocat Behav. 1994;45:79-122.

20. Lent RW, Brown SD, Larkin KC. Self-efficacy in the prediction of academic performance and perceived career options. J Couns Psychol. 1986;33(3):265-269.

21. Locke EA, editor. Handbook of principles of organizational behavior. Oxford, UK: Blackwell; 2000.

22. Locke EA, Latham GP. Building a practical useful theory of goal setting and task motivation. A 35-year odyssey. Am Psychol. 2002; 57(9):705-717.

23. Pajares F. Self-efficacy beliefs in academic settings. Rev Educ Res. 1996;66(4):543-578.

24. Pajares F. Overview of social cognitive theory and of self-efficacy. [Internet]. Lexington, KY: P20 Motivation and Learning Lab; 2002. [cited 2009 Oct 15]. Available from: http://www.emory.edu/EDUCATION/mfp/eff.html

25. Pajares F, Schunk DH. Self-beliefs and school success: Self-efficacy, self-concept, and school achievement. In R. Riding \& S. Rayner, editors. Perception. London: Ablex Publishing; 2001.

26. Pasupathy R, Bogschutz RJ. An investigation of graduate speechlanguage pathology students' SLP clinical self-efficacy. Contemp Issues Commun Sci Disord. 2013;40:151-159.

27. Rudolf S, Manning WH, Sewell WR. The use of self-efficacy scaling in training student clinicians: Implications for work with stutterers. J Fluency Disord. 1983;8:55-75.

28. Schunk DH. Sequential attributional feedback and children's achievement behaviors. J Educ Psychol. 1984;76:1159-1169.

29. Usher EL, Pajares F. Sources of self-efficacy in school: Critical review of the literature and future directions. Rev Educ Res. 2008;78 751-796.

30. Wood R, Bandura A. Social cognitive theory of organizational management. Acad Manage Rev. 1989;14(3):361-384. 